\title{
KM3NeT Time Calibration
}

\author{
Irene Di Palma ${ }^{1, *}$ \\ ${ }^{1}$ Universitá di Roma La Sapienza and Istituto Nazionale di Fisica Nucleare, \\ Sezione di Roma, Italy, Piazzale Aldo Moro 2, 00185 Roma, Italy
}

\begin{abstract}
The KM3NeT detectors are large three-dimensional arrays of several thousands digital optical modules under construction in the Mediterranean Sea. The basic detection element of the neutrino telescope is the digital optical module containing 31 three-inch photomultiplier tubes. Each detection unit, composed of 18 digital optical modules, is a mechanical structure anchored to the sea floor, held vertical by a submerged buoy for the detection of Cherenkov light emitted by charged secondary particles emerging from neutrino interactions. Detector calibration, i.e. timing, positioning and sea-water properties, is overviewed in this talk and discussed in detail in this conference.
\end{abstract}

\section{The KM3NeT Detector}

KM3NeT belongs to a novel generation of under water neutrino telescopes [1]. The infrastructure will consist of three so-called building blocks, each made of 115 lines (or detector units DU) of 18 optical modules, that have 31 photo-multiplier tubes each. KM3NeT is made of KM3NeT/ORCA (Oscillation Research with Cosmics in the Abyss) in Toulon, France and KM3NeT/ARCA (Astroparticle Research with Cosmics in the Abyss) in Capo Passero, Sicily. The main objectives of KM3NeT are the discovery and subsequent observation of high-energy neutrino sources in the Universe and the determination of the neutrino mass hierarchy. Neutrinos can interact with matter inside or in the vicinity of the detector producing secondary particles that can be detected through the Cherenkov light that they produce. Due to the long range in water, the conventional detection channel is given by muons produced in charged current interactions of muon neutrinos. Furthermore, KM3NeT will have significant sensitivity to all the neutrino interactions.

The basic detection element of the neutrino telescope is the digital optical module (DOM), a 17-inch pressure resistant glass sphere containing 31 3-inch photomultiplier tubes (PMTs), a number of calibration devices and the read-out electronics. The multi-PMT design provides a large photocathode area, good separation between single-photon and multiple-photon hits and information on the photon direction. DOMs are arranged by modulo eighteen on the flexible lines attached to the sea bottom. Each line has a base module close to the anchor which performs the line power control and the optical link signal amplification. The detector is connected to the shore via the main electro-optical cable. The single fiber in the cable (about 40 $\mathrm{km}$ in Toulon, and $100 \mathrm{~km}$ in Capo Passero) is common for the base and the DOMs. Each DOM is an IP node in an Ethernet network. The information recorded from a PMT consists of the start time and the Time over Threshold (ToT). The start time is defined as the time at

\footnotetext{
*e-mail: Irene.DiPalma@roma1.infn.it
} 
which the pulse passes beyond a 0.3 p.e. threshold and the ToT is the time the pulse remains above this threshold. Onshore, the physics events are filtered from the background by an online trigger algorithm and stored on disk. Data collected by the PMTs are digitised in the DOMs and sent to shore, where they are filtered by appropriate triggering algorithms. Accurate measurements of the light arrival times and charges and precise real-time knowledge of the positions and orientations of the PMTs are required for the accurate reconstruction of the direction of the secondary particles.

DOMs move under the effects of underwater currents, these movements are continuously monitored through an underwater positioning system based on acoustic emitters and receivers. In fact, each DOM is equipped with an internal acoustic piezo detector for the acoustic positioning system [2], while each line base is equipped with an external hydrophone which has better sensitivity compared to the piezo detectors to reference the line position on the sea bed. The acoustic signal transit time is measured periodically and for three or more emitters position of the receiver can be measured with trilateration. The acoustic emitters are installed in the calibration units that will be placed at known positions on the sea bed. In addition, each calibration unit contains a laser beacon (composed of a piezo-ceramic transducer and an integrated electronics board) for interline time calibration and water optical properties monitoring, a long-baseline acoustic beacon for DOMs positioning, hydrophone for their own positioning, and environmental monitoring instruments (conductivity, salinity, temperature, sound velocity, sea currents). The DOM orientation is measured thanks to the internal attitude and heading reference system board.

\section{Time Calibration}

For the neutrino event reconstruction with a precision better than $1^{\circ}$, the optical modules need to be synchronized with sub-nanosecond precision and their position determined with better than meter precision. Each DOM and the base have identical Control Logic Boards which perform the signal processing and transfer, time synchronization and control of the instruments. Time synchronization between different detector components is monitored in situ by light propagation time measurements between light emitters (LED nanobeacons and lasers) and PMTs.

The time calibration at a nanosecond level is necessary to achieve the envisaged muon track angular resolution for a neutrino telescope. For this, the following time offsets have to be determined: 1) Intra-DOM; 2) Inter-DOM; 3) Inter-DU; 4) nanobeacons. Prerequisite for the time calibration is the PMT characterization through the High-Voltage (HV) tuning: all the PMTs in the DOM have to be set to the same gain value. The high voltage tuning is based on the estimation of the ToT duration value of a single detected photon. For this purpose, background runs are used. For one PMT of one DOM, all hits detected during the run are read. A hit is composed of a time stamp for timing purpose and of a ToT duration, for light intensity purpose. The tuning is done with $25 \mathrm{~V}$ steps of $\mathrm{HV}$ and ToT is calculated by means of a Gaussian fit function to the ToT distribution during $1 \mathrm{~min}$ of data taking for each step. The proper HV, corresponding to ToT $26.4 \mathrm{~ns}$, is found from a Gaussian fit to ToT vs HV plot, see Figure 1. This ToT value corresponds to the average ToT value estimated on a subset of PMTs with properly calibrated gain $\left(3 \times 10^{6}\right)$.

\subsection{Intra-DOM calibration}

The Intra-DOM time offsets between PMTs in the same DOM primarily depend on the PMT transit time. Radioactive potassium decay in the glass and the gel can be simultaneously seen 


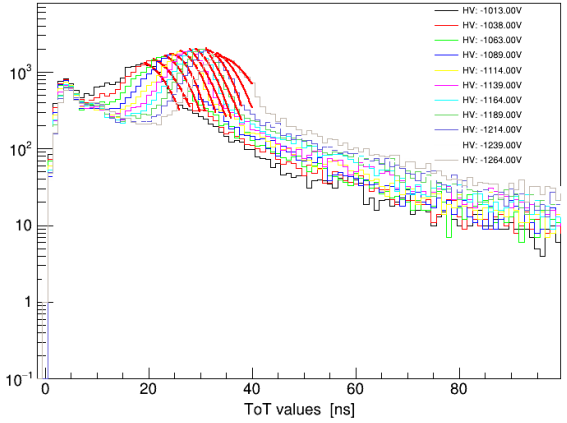

Figure 1. Example of ToT histograms and the corresponding Gaussian fit for PMT\#5 of DOM\#3 of string\#1 and for several high voltage offset values. The tuning is done with $25 \mathrm{~V}$ steps of $\mathrm{HV}$ and ToT is calculated by means of a Gaussian fit function to the ToT distribution during $1 \mathrm{~min}$ of data taking for each step. The average ToT value, estimated on a subset of PMTs with properly calibrated gain $\left(3 \times 10^{6}\right)$, corresponds to $26.4 \mathrm{~ns}$.

by the neighbour PMTs in the DOM, and can produce up to 150 Cherenkov photons per decay [3]. These decays are the main source of the single PMT rate. A single decay occurring in the vicinity of the DOM has a chance to produce a genuine coincidence between signals of different PMTs, which can be exploited for time calibration of the DOM. This feature is used to verify the PMT mapping and to perform inter-PMT time calibration.

The distributions of time differences between signals detected in different PMTs in the same DOM are studied as a function of the angular separation of the PMTs involved. The distribution of hit time differences between all possible combinations of PMT pairs are assumed to follow a Gaussian shape. For each DOM with $N=31$ PMTs, a total of $N(N-1) / 2$ distributions are produced and shown in Figure 2 (left) for DOM 1. In the figure, the numbers of PMT pairs are ordered according to their angular separation. The correlation peak decreases as the angular separation increases due to the limited field of view of each PMT, see Figure 2 (right). These distributions are well fitted by a Gaussian function. The mean values, heights and widths of the Gaussian peaks are related to the time offsets, detection efficiencies and intrinsic time-spreads of all the PMTs. Typically, a FWHM of 7-10 ns is found for all different PMT pairs, mostly reflecting the intrinsic PMT transit time spread of up to $5 \mathrm{~ns}$ at FWHM.

\subsection{Inter-DOM calibration}

The Inter-DOM time offsets, between DOMs, primarily depend on the cable lengths. The delay of the laser signal is measured for each DOM to perform the line time calibration. The delay of about $200 \mathrm{~ns}$ between DOMs corresponds to about $40 \mathrm{~m}$ of the optical fiber connecting the DOMs, while a delay of about $750 \mathrm{~ns}$ is the time difference between the base and DOM1 due to the length of the optical fiber and the all samples' time delays. The total delay for the light detection by reference PMTs of each DOM is calculated using the known laser system propagation time and the cable length connecting the line to the test shore station. After the deployment, this delay will be increased by the known delay introduced by the underwater infrastructure. No time offset shifts were observed within constantly powered periods. The values obtained with this procedure are stable to within a few nanoseconds over a stable period of data taking.

\subsection{Inter-DU calibration}

The Inter-DU calibration is based on the measurement of the Round-Trip-Time (RTT) delay of the laser signal between the reference clock (called master) and the DU base, due to the length of the optical fiber $(\sim 100 \mathrm{~km})$. We found constant time difference between DU1 and DU2, due to the length difference between the DUs inter-link cables $(\sim 100 \mathrm{~m} \times 2)$. 

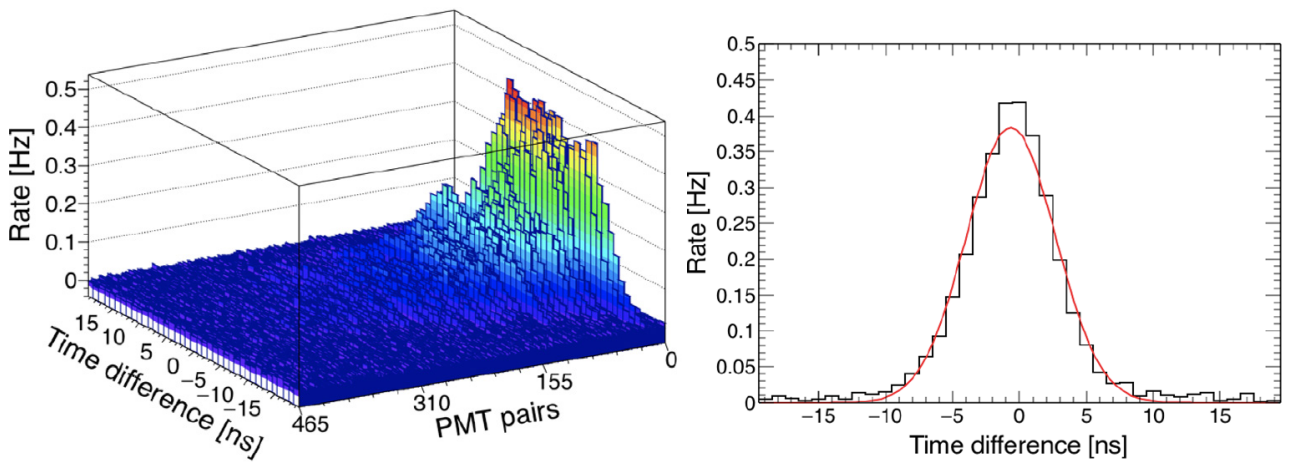

Figure 2. Left: distribution of time differences between the hit times for all PMT pairs in DOM 1 for one physics run. The PMT pairs are ordered according to the angular distance between PMTs. Right: distribution of time differences between the hit times of two adjacent PMTs in DOM 1 for one physics run. The Gaussian function, represented by the red line, is the result of the simultaneous fit. The baseline due to combinatorial background has been subtracted from the data. [1].

\section{Calibration after deployment}

Several instruments will be used to perform the verification and adjustment of the time calibration in- situ. ${ }^{40} \mathrm{~K}$ decays in water will be used for PMT time delay calibration between PMTs in the same DOM. The nanobeacons installed in each DOM provide light detection from one DOM to the neighbor DOMs for their inter calibration. Vertical atmospheric muons provide alternative inter-DOM calibration. Laser beacons installed on the Calibration Units provide light detection by DOMs of different lines for the time calibration between the lines. Positioning of the DOMs will be performed thanks to the acoustic instruments installed in the DOMs, DU bases and calibration units. To monitor the time calibration after deployment we will use atmospheric muons, atmospheric neutrinos, laser beacons and nanobeacons.

\subsection{Nanobeacons}

Each DOM is equipped with a remotely controlled flashable LED (nanobeacon), installed on the top of the DOM, pointing upward to DOMs higher in the DU. The difference between the time of the detection of the LED light and the emission time of the light by the LED is monitored, by using the PMT time offsets obtained from the dark room and taking into account the travel time of the light in the medium. For more details see [4]

\section{Summary}

The KM3NeT detector is under construction. The procedure for time calibration exploiting potassium decays and LED beacons was demonstrated successfully with nanosecond stability during first DU line integration.

\section{References}

[1] S. Adrian-Martinez et al., J.Phys. G43, no.8, 084001 (2016).

[2] S. Viola, F. Simeone and M. Saldana, EPJ Web of Conferences 116, (2016).

[3] J.A. Aguilar et al., Astropart. Phys. 33, 86 (2010).

[4] D. Calvo, talk given at this conference. 